\title{
Drift-free Attitude Estimation Using Quasi-linear Observers
}

\author{
Henrik Rehbinder ${ }^{1}$ and Xiaoming $\mathrm{Hu}^{2}$ \\ 1 RaySearch Laboratories, Sweden henrik.rehbinder@raysearchlabs.com \\ 2 Optimization and Systems Theory, Royal Institute of Technology, Sweden \\ hu@kth.se
}

Summary. In this paper we study the attitude estimation problem for an accelerated rigid body using gyros and accelerometers. The application in mind is that of a walking robot and particular attention is paid to the large and abrupt changes in accelerations that can be expected in such an environment. We propose a state estimation algorithm that fuses data from rate gyros and accelerometers to give long-term drift free attitude estimates. The algorithm does not use any local parameterization of the rigid body kinematics and can thus be used for a rigid body performing any kind of rotations. The algorithm is a combination of two non-standard, but in a sense linear, Kalman filters between which a trigger based switching takes place. The kinematics representation used makes it possible to construct a linear algorithm that can be shown to give convergent estimates for this nonlinear problem. The state estimator is evaluated in simulations demonstrating how the estimates are long term stable even in the presence of gyrodrift.

\subsection{Introduction}

A prerequisite for mobile robot control is state estimation where the states typically are position, velocity and orientation. State estimation is especially important for walking robots in difficult terrain where a sense of balance is absolutely necessary as it is the basis of attitude control. With attitude we refer to the robot's orientation relative to the gravity vector, usually described by pitch and roll. Perhaps most important to walking robots, the problem still applies to any kind of robot moving in difficult terrain. Attitude estimation is usually performed by combining measurements from three kinds of sensors: rate gyros, inclinometers and accelerometers. It is possible to use a rate gyro to derive attitudes by integrating the rigid body kinematic equations. With high quality gyros and good initial values these estimates can be very accurate over long periods of time. However, if the aim is an autonomous vehicle then the attitude estimate should be reliable over an infinite time scale. It would also be desirable to use cheap gyros as high quality gyros are expensive. To provide an absolute reference of the attitude, inclinometers and accelerometers 
which relate the body to the gravity vector can be used. A problem is that both these sensors also are sensitive to translational accelerations. The sensor errors described are complementary in terms of frequency characteristics. Due to this complementarity, the estimation errors can be decreased by sensor fusion [1], [26]. If the rigid body motion is restricted to planar rotation, it would be possible to formulate the sensor fusion problem as a linear observer problem which can be solved by standard Kalman filtering techniques. Fundamental for walking robots is that the body motion is inherently three-dimensional, making most kinematics representations nonlinear. For nonlinear problems, there are no general state estimation algorithms that are guaranteed to work. Attitude estimation via different ensembles of the above mentioned sensors has been studied by many authors such as Baerveldt and Klang [2], Balaram [3], Barshan and Durrant-Whyte [4], Foxlin [8], [9], Greene [10] Lefferts et. al [13], Madni [14], Rehbinder and Hu [17], [18], Sakaguchi et. al [21], Smith et. al [22] and Vaganay et.al [25].

In this paper we provide a solution to fusing data from a 3-axis rate gyro and a 3-axis accelerometer that will provide stable estimates of the robot's attitude. By using a global description of rigid body rotation we are able to obtain a linear problem and can use a modified linear Kalman filter. Further, we show how the linear Kalman filter can be used to obtain a projected Kalman filter, where the states evolve on the unit sphere, a curved space. The global kinematics representation used eliminates all the nonlinear problems associated with Euler angles in a remarkably elegant and simple way. This is paid for by a slight increase in abstraction. As the kinematics representation used is global, there are no restrictions on the kind of motion the robot is allowed to perform, contrary to what would be the case if for example Euler angles were used. The projected Kalman filter can be given a geometric interpretation which is related to the observer presented by Rehbinder and Ghosh in [16], [15] where an observer for an implicit output system evolving on $S O(3)$ is presented. It would be possible to formulate large parts of this paper in a strict differential geometric framework but for the sake of readability, we choose not to do so. Further we discuss how to modify the observer gains in order to take accelerations that are not well modeled as white noise into account.

The main contributions of this article are: Two mathematically sound, quite simple algorithms that solves the important problem of fusing accelerometer and gyro data; The use of a global kinematics representation and a design idea for observers evolving on a curved space; An experimental evaluation of the observers, using a walking robot. The outline of the paper is as follows. In Section 1.2 the problem is formulated and the proposed algorithms are described and analyzed. 


\subsection{Problem formulation and solution}

As is well known, rigid body kinematics have a somewhat delicate structure as rotations are most naturally described as elements of the rotation group $S O(3)$, a smooth manifold. It is common practice to consider some parameterization of $S O(3)$ such as various versions of Euler angels or quaternions. In this study we will work directly with the rotation matrix representation of $S O(3)$. We embed the manifold in a larger linear space and it is possible to obtain a linear problem formulation that is used for the observer design. Further, the kinematics representation is global, as opposed to the local Euler coordinates.

Apart from the above, the problem of distinguishing between inertial forces and the gravity vector is discussed. We will use an accelerometer as the at titude sensor. When the body is accelerated, which is the case for walking robots, the accelerometer noise can be very high. The straightforward Kalman filtering way of handling this problem is to assign a large covariance matrix to the output noise. We will investigate an approach where a variable output noise covariance is used. This variable noise covariance is designed to compensate for the variations in acceleration magnitude that originates from the feet impacts.

\subsubsection{Mathematical modeling}

Consider a rigid body moving in inertial space. The body is undergoing both rotations and translations and our aim is to estimate attitude, the robots orientation relative to the gravity vector. For this, a 3-axis rate gyro and a 3 axis accelerometer is used. Introduce a coordinate system, $\mathrm{N}$, fixed in inertial space and a coordinate system, B, fixed in the body. Let the coordinates of an arbitrary point $\xi$ be denoted by $\xi^{N}$ if expressed in the $N$-frame and by $\xi^{B}$ if expressed in the $B$-frame. The relation between the two frames is

$$
\xi^{B}=R\left(\xi^{N}-r^{N}\right)
$$

where $R$ is a rotation matrix, that is

$$
R \in S O(3)=\left\{R \in \mathbb{R}^{3 \times 3}: R^{\prime} R=I, \operatorname{det} R=1\right\} .
$$

The kinematics of a rigid body are

$$
\left\{\begin{array}{l}
\ddot{r}^{N}=a \\
\dot{R}=\Omega(t) R
\end{array}\right.
$$

where $a$ is the acceleration expressed in the $N$-frame and where

$$
\Omega=\left(\begin{array}{ccc}
0 & \omega_{3} & -\omega_{2} \\
-\omega_{3} & 0 & \omega_{1} \\
\omega_{2} & -\omega_{1} & 0
\end{array}\right)
$$


$\omega_{i}$ are the components of the angular velocity expressed in the $B$-frame. The matrix $\Omega$ has the property that $\Omega x=-\omega \wedge x$ and is therefore called the wedge matrix. We consider the body to be equipped with a strap-down inertial measurement unit (IMU), that is, a body fixed rate-gyro/accelerometer package. The rate gyro measures the angular velocity in the body frame up to an unknown and slowly varying offset. In the analysis and observer design we will neglect this offset and therefore we take the angular velocity as a known entity in the problem.

The strap-down accelerometer measures the difference between the inertial forces and gravity, expressed in the body frame $B$. If the accelerometer output is denoted $y$, then the accelerometer model is

$$
y=R\left(a-g^{N}\right) .
$$

From (1.5) it is obvious that the accelerometer may be used as an attitude sensor if the acceleration itself is considered as a disturbance and the gravity vector as the entity that we want to measure. As a matter of fact, attitude may be defined as the body's orientation relative to the gravity vector. It is perhaps more standard to consider attitude as being described by the two Euler angles pitch and roll. The Euler angle formulation of the problem is here reviewed for completeness and comparison. In the yaw $(\psi)-\operatorname{pitch}(\theta)-\operatorname{roll}(\phi)$ parameterization of $S O(3)$

$$
R=\left(\begin{array}{ccc}
\mathrm{c} \psi \mathrm{c} \theta & \mathrm{s} \psi \mathrm{c} \theta & -\mathrm{s} \theta \\
-\mathrm{s} \psi \mathrm{c} \theta+\mathrm{c} \psi \mathrm{s} \theta \mathrm{s} \phi & \mathrm{c} \psi \mathrm{c} \phi+\mathrm{s} \psi \mathrm{s} \theta \mathrm{s} \phi & \mathrm{c} \theta \mathrm{s} \phi \\
\mathrm{s} \psi \mathrm{s} \phi+\mathrm{c} \psi \mathrm{s} \theta \mathrm{c} \phi & \mathrm{s} \psi \mathrm{s} \theta \mathrm{c} \phi-\mathrm{c} \psi \mathrm{s} \phi & \mathrm{c} \theta \mathrm{c} \phi
\end{array}\right),
$$

where $\mathrm{c} \psi=\cos \psi$ etc. As $g^{N}=-g e_{3}$ where $g=9.81 \mathrm{~m} / \mathrm{s}^{2}$, and where $e_{3}$ is the third unit vector, the accelerometer output may be written

$$
y=\left(\begin{array}{c}
-\sin \theta \\
\cos \theta \sin \phi \\
\cos \theta \cos \phi
\end{array}\right) g+R a
$$

The pitch-roll kinematics can be derived by straightforward differentiation of (1.6) and (1.3) and are

$$
\left(\begin{array}{c}
\dot{\theta} \\
\dot{\phi}
\end{array}\right)=\left(\begin{array}{ccc}
0 & \cos \phi & -\sin \phi \\
1 & \sin \phi \tan \theta & \cos \phi \tan \theta
\end{array}\right)\left(\begin{array}{l}
\omega_{1} \\
\omega_{2} \\
\omega_{3}
\end{array}\right) .
$$

Therefore, the attitude kinematics and measurements may be written as the nonlinear system

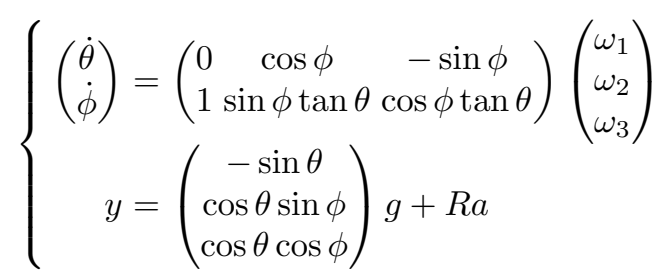


This system is nonlinear, both in the dynamics and in the output equation. Further, the yaw-pitch-roll parameterization is not one-to-one globally and clearly $\dot{\theta}$ and $\dot{\phi}$ are undefined for $\theta= \pm \pi / 2$. This makes the representation unsuitable for general robots. It would for example not be possible to use (1.9) for a climbing robot which operates around $\phi= \pm \pi / 2$. Further, it is far from easy to design a convergent observer for (1.9) due to the nonlinearities. The observer design problem is further complicated by that $\dot{\phi} \rightarrow \infty$ when $|\theta| \rightarrow \pi / 2$.

Consider instead the possibility of using the rotation matrix itself as state. This will in a remarkably clear way eliminate most of the problems associated with (1.9). The rotational kinematics $\dot{R}=\Omega(t) R$ may be written column-wise as

$$
\dot{r}_{i}=\Omega(t) r_{i}, \quad i=1,2,3
$$

where $r_{i}$ is the $i^{\text {th }}$ column of $R$. In the same way, the accelerometer output can be written

$$
y=r_{3} g+R a .
$$

Denote now by

$$
\begin{aligned}
& x=r_{3} \\
& u=a / g,
\end{aligned}
$$

and with a slight abuse of notation

$$
y:=y / g .
$$

In this notation, the attitude kinematics and accelerometer measurements is described by the differential-algebraic equation

$$
\left\{\begin{array}{l}
\dot{x}=\Omega(t) x \\
\|x\|-1=0 \\
y=x+w
\end{array}\right.
$$

where $w=R u$. The algebraic constraint $\|x\|^{2}-1=0$, which captures that $x$ is a rotation matrix column, is better viewed as the geometric constraint

$$
x \in \mathcal{S}^{2}=\left\{x \in \mathbb{R}^{3}:\|x\|^{2}-1=0\right\},
$$

where $\mathcal{S}^{2}$ is the unit sphere. As a matter of fact, $\mathcal{S}^{2}$ is a manifold of a special kind, a smooth hypersurface embedded in $\mathbb{R}^{3}$. It is well known that $\mathcal{S}^{2}$ is an invariant set under $\dot{x}=\Omega(t) x$, so the algebraic constraint can be replaced by an initial value constraint. The system (1.14) can therefore be written

$$
\left\{\begin{array}{l}
\dot{x}=\Omega(t) x \\
x(0)=x_{0} \in \mathcal{S}^{2} \\
y=x+w
\end{array} .\right.
$$

There is an appealing linear structure to this formulation, but, note that the vector field $\Omega(t) x$ viewed as a mapping $\kappa_{t}: \mathcal{S}^{2} \rightarrow \mathbb{R}^{3}$ defined by 


$$
\kappa_{t}: x \mapsto \Omega(t) x
$$

is not linear. Linearity is only defined between linear spaces and $\mathcal{S}^{2}$ is not a linear space. However, if $\kappa_{t}$ is extended to $\mathbb{R}^{3}, \kappa_{t}: \mathbb{R}^{3} \rightarrow \mathbb{R}^{3}$, by the same definition (1.17) then it may be viewed as a linear mapping. This is perhaps not a very surprising observation but it still is the key insight needed to formulate the simple observers we will present.

The constraint $x \in \mathcal{S}^{2}$ adds a difficulty to the observer problem. It must be ensured that the state estimates $\hat{x} \in \mathcal{S}^{2}$. If this is not ensured, $\hat{x}$ can not be the column of a rotation matrix. However, the problem formulated as in (1.16) is still much easier to solve than formulated in the form (1.9). It will be shown that it is possible to base the observer design on the embedded linear system

$$
\left\{\begin{array}{l}
\dot{x}=\Omega(t) x \\
x(0)=x_{0} \in \mathbb{R}^{3} \\
y=x+w
\end{array} .\right.
$$

and still obtain state estimates $\hat{x} \in \mathcal{S}^{2}$.

\subsubsection{Two linear Kalman filter based observers}

Consider the system model (1.18). It is linear and clearly it is observable as the entire state vector is measured. Therefore, the standard deterministic linear Kalman filter [24]

$$
\dot{z}=\Omega(t) z+L(t)(y-z)
$$

where

$$
\begin{aligned}
& L(t)=P(t) Q_{2}^{-1} \\
& \dot{P}=\Omega(t) P+P \Omega(t)^{\prime}+Q_{1}(t)-P Q_{2}(t)^{-1} P
\end{aligned}
$$

is an exponentially convergent observer for properly chosen $Q_{1}(t), Q_{2}(t)$. When using the deterministic framework, the matrices $Q_{1}$ and $Q_{2}$ have no interpretation as noise covariances. However, the usual heuristics that large measurement errors calls for a large $Q_{2}$ and that large system uncertainties calls for large $Q_{1}$ still applies. Now, (1.19) can actually be used to estimate $x \in \mathcal{S}^{2}$. In the noise free case, $\|z(t)-x(t)\| \rightarrow 0$ as $t \rightarrow \infty$ so $z(t) \rightarrow \mathcal{S}^{2}$. For finite $t$, and in the presence of sensor noise, there are no guarantees that $z(t) \in \mathcal{S}^{2}$. But, this problem is solved easily by adding a projection $P_{\mathcal{S}^{2}}$ of $z$ onto $\mathcal{S}^{2}$, outside the observer dynamics (1.19). The projection onto $\mathcal{S}^{2}$ is of course very straightforward

$$
P_{\mathcal{S}^{2}} z=\frac{z}{\|z\|}
$$

if we assume that $z \neq 0$.

Remark 1. When implementing (1.23) in discrete time, the problem of handling $z=0$ in (1.21) is easily solved by instead using

$$
\hat{x}(t)=\left\{\begin{array}{l}
z(t) /\|z(t)\|, \quad \text { if }\|z(t)\| \neq 0 \\
\hat{x}(t-1), \quad \text { if }\|z(t)\|=0
\end{array} .\right.
$$


The observer

$$
\left\{\begin{array}{l}
\dot{z}=\Omega(t) z+L(t)(y-z) \\
\hat{x}=P_{\mathcal{S}^{2}} z
\end{array}\right.
$$

will hereafter be referred to as the pseudo-linear Kalman filter. An illustration of the idea behind the pseudo-linear Kalman filter is given in Figure 1.1 where it is shown how the states $x$ and the state estimates $\hat{x}$ evolve on the sphere while the observer states $z$ evolve in $\mathbb{R}^{3}$. It must be pointed out that the reason

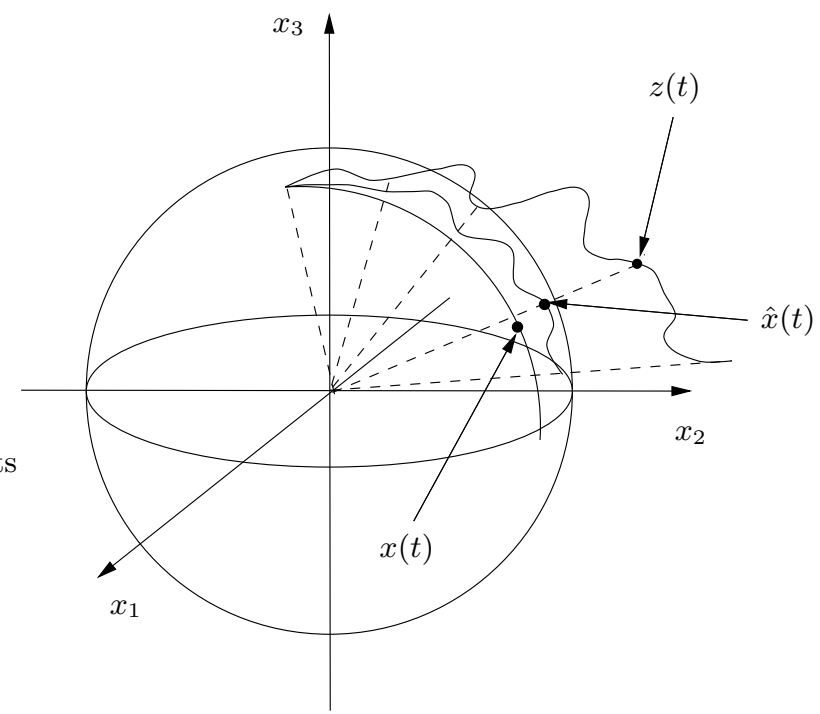

Fig. 1.1. Pseudo-linear Kalman filter states $z$ and state estimates $\hat{x}$ along with true states $x$.

why this very simple observer works is that the system (1.16) is observable, without the information that $\hat{x}(0) \in \mathcal{S}^{2}$, that is, that (1.18) is observable. If, for example, the accelerometer was 2-axis, then the system (1.18) would not be observable and some other method would have to be used.

The idea presented here was to use an observer where the states did not evolve on $\mathcal{S}^{2}$ but anyway converged and then project the observer states onto $\mathcal{S}^{2}$. It would also be possible to design an observer where the states evolve on $\mathcal{S}^{2}$, just as for the underlying system. For an observer to evolve on $\mathcal{S}^{2}$, it must be so that the state estimate derivatives are tangential to the sphere, that is, that

$$
\dot{\hat{x}} \in T_{\hat{x}} \mathcal{S}^{2}
$$

where

$$
T_{\hat{x}} \mathcal{S}^{2}=\left\{x \in \mathbb{R}^{3}: \hat{x}^{\prime} x=0\right\}
$$


is the tangent space of the sphere at the point $\hat{x}$. The observer (1.23) can be used to design such an observer. If the observer vector field is projected orthogonally onto $T_{\hat{x}} \mathcal{S}^{2}$, then an observer evolving on $\mathcal{S}^{2}$ is obtained. We note that the orthogonal projection onto $T_{\hat{x}} \mathcal{S}^{2}$ is given by

$$
P_{T_{\hat{x}} \mathcal{S}^{2}}=\left(I-\hat{x} \hat{x}^{\prime}\right)
$$

Proof: It is well known that $T_{\hat{x}} \mathcal{S}^{2}=$ ker $\hat{x}^{\prime}$, the orthogonal complement of $\{\hat{x}\}$. As ker $\hat{x}^{\prime}=(\operatorname{Im} \hat{x})^{\perp}$ and as $\|\hat{x}\|=1$, it is clear that $P_{T_{\hat{x}} \mathcal{S}^{2}}=\left(I-\hat{x} \hat{x}^{\prime}\right) . \square$ The projection of the vector field is

$$
P_{T_{\hat{x}} \mathcal{S}^{2}}(\Omega(t) \hat{x}+L(t)(y-\hat{x}))=\Omega(t) \hat{x}+\left(I-\hat{x} \hat{x}^{\prime}\right) L(t)(y-\hat{x})
$$

due to that $\Omega(t) \hat{x} \in T_{\hat{x}} \mathcal{S}^{2}$. We can formulate the projected Kalman filter, defined by

$$
\left\{\begin{array}{l}
\dot{\hat{x}}=\Omega(t) \hat{x}+\left(I-\hat{x} \hat{x}^{\prime}\right) L(t)(y-\hat{x}) \\
\hat{x}(0)=x_{0} \in \mathcal{S}^{2}
\end{array}\right.
$$

with the property that $\hat{x}(t) \in \mathcal{S}^{2} \forall t$. The projected Kalman filter can actually be shown to be convergent for a special choice of filter parameters. The proof of this is particularly simple if a natural error measure for this geometric setting is used. This error measure is based on the scalar product and is a simplified version of the error measure introduced in [12] for states on $S O(3)$. We note that $\xi=1-x^{\prime} \hat{x}$ is an error measure for (1.28) in the sense that $\xi=0 \Leftrightarrow x=\hat{x}$. Further $0 \leq \xi \leq 2$.

Proof: As $x$ and $\hat{x}$ are of unit length, $x^{\prime} \hat{x}=\cos \alpha$ for some $\alpha \in[0,2 \pi)$ and the proof readily follows.

Theorem 1. Let $L(t)=l(t) I$ and let $l(t)$ be continuous, non-negative and bounded from above. Further, let there be $T<\infty$ and $\delta>0$ such that for every $t$

$$
\int_{t}^{t+T} l(\tau) d \tau \geq \delta
$$

Then the error dynamics of the projected Kalman filter (1.28) is exponentially stable and $0 \leq \xi<2$ is contained in the domain of attraction. The only feasible $\xi$ for which the error dynamics are unstable is $\xi=2$ which corresponds to $x(0)=-\hat{x}(0)$.

Proof: Consider the nominal dynamics of the error $\xi=1-x^{\prime} \hat{x}$ where the noise is set to be zero.

$$
\begin{aligned}
\dot{\xi} & =-\dot{x}^{\prime} \hat{x}-x^{\prime} \dot{\hat{x}}=-x^{\prime}\left(\Omega(t)+\Omega(t)^{\prime}\right) \hat{x}-l(t) x^{\prime}\left(I-\hat{x} \hat{x}^{\prime}\right)(x-\hat{x}) \\
& =-l(t) x^{\prime}\left(I-\hat{x} \hat{x}^{\prime}\right) x=-l(t)\left(1-x^{\prime} \hat{x} \hat{x}^{\prime} x\right) \\
& =-l(t) \xi(2-\xi) .
\end{aligned}
$$

Since (1.30) is a Bernoulli equation, the solution can be written 


$$
\xi(t)=\frac{\xi_{0} e^{-2 \int_{t_{0}}^{t} l(s) d s}}{\left(1-\frac{\xi_{0}}{2}\right)+\frac{\xi_{0}}{2} e^{-2 \int_{t_{0}}^{t} l(s) d s}}
$$

Consequently,

$$
|\xi(t)| \leq \frac{\left|\xi_{0}\right|}{\left(1-\frac{\left|\xi_{0}\right|}{2}\right)} e^{-2 \int_{t_{0}}^{t} l(s) d s}
$$

and by noting that from (1.29)

$$
\int_{t_{0}}^{t} l(s) d s \geq \frac{\delta}{2 T}\left(t-t_{0}\right), \quad t \geq t_{0}+T
$$

it is proven that $(1.28)$ is exponentially stable and that $[0,2)$ is contained in the domain of attraction.

Remark 2. The gain matrix $L(t)=l(t) I$ does not have to be a Kalman gain for Theorem 1 to hold. Further, the integral constraint (1.29) does not exclude the possibility that $l(t)=0$ on intervals. This makes it possible to turn off the accelerometer during phases when the acceleration is extremely high and still obtain convergent estimates.

For the following simple set of Kalman filter parameters, $L(t)=l(t) I$ and the projected Kalman filter converges.

Corollary 1. Let $P_{0}=p_{0} I, Q_{1}(t)=q_{1}(t) I, Q_{2}(t)=q_{2}(t) I$ and let there be $\underline{q}_{1}>0, \bar{q}_{1}<\infty, \underline{q}_{2}>0, \bar{q}_{2}<\infty$ such that $\underline{q}_{1} \leq q_{1}(t) \leq \bar{q}_{1}$ and $\underline{q}_{2} \leq q_{2}(t) \leq \bar{q}_{2}$. Let further $q_{1}(t)$ and $q_{2}(t)$ be continuous functions. Then the conditions in Theorem 1 are fulfilled.

Proof: Take $P(t)=p(t) I$ and show that $P(\tau)=p(\tau) I$ for $\tau \geq t$.

$$
\dot{P}(t)=p(t)\left(\Omega(t)+\Omega(t)^{\prime}\right)+q_{1}(t) I-\frac{p(t)^{2}}{q_{2}(t)} I=\left(q_{1}(t)-\frac{p(t)^{2}}{q_{2}(t)}\right) I
$$

as $\Omega(t)=-\Omega(t)^{\prime}$. As $P(0)=p_{0} I, P(t)=p(t) I \forall t$. From stochastic observability and reachability and the standard Riccati equation theory [6] it follows that $\exists l_{l}>0$ and $l_{u}<\infty$ such that $l_{l} \leq l(t)<l_{u}$. Therefore (1.29) is fulfilled.

In our experiment the two observers are found to have similar performances. The question if there are any conditions under which the two observers actually are equivalent may therefore be asked. The answer is that there is such a condition and that it is very special. However, it is in an approximate sense fulfilled in the experiments and therefore we discuss it here.

Remark 3. If $y=\gamma(t) \hat{x}$ then the two observers (1.23) and (1.28) are equivalent in the sense that

$$
\{(z, \hat{x}): z /\|z\|-\hat{x}=0\}
$$

is an invariant set under the dynamics of the two observers 


$$
\left\{\begin{array}{l}
\dot{z}=\Omega(t) z+l(t)(y-z) \\
\hat{\hat{x}}=\Omega(t) \hat{x}+l(t)\left(I-\hat{x} \hat{x}^{\prime}\right)(y-\hat{x})
\end{array} .\right.
$$

Proof: $z /\|z\|-\hat{x}=0$ can equivalently be written $h(z, \hat{x})=z-\|z\| \hat{x}=0$. By straightforward differentiation,

$$
\frac{d}{d t} h(z, \hat{x})=l(t)(1+\|z\|)\left[y-\hat{x}\left(\hat{x}^{\prime} y\right)\right]
$$

so for $h(z, \hat{x})=0$ to be invariant it must hold that

$$
y=\gamma(t) \hat{x}
$$

for some $\gamma(t)$.

\subsubsection{A geometric interpretation}

The projected Kalman filter was derived by taking the standard Kalman filter and projecting the observer vector field on $T_{\hat{x}} \mathcal{S}^{2}$. It turns out that the projected Kalman filter, for $L(t)=l(t) I$, can be given a quite precise geometric interpretation which clearly illustrates the observer dynamics. For any system $\dot{x}=f(x, t)$ evolving on $\mathcal{S}^{2}$, the vector field must be such that $x^{\prime} f(x, t) \forall x \in \mathcal{S}^{2}, \forall t$ or in other words that $f(x, t) \in T_{\hat{x}} \mathcal{S}^{2}$. A spanning set for $T_{\hat{x}} \mathcal{S}^{2}$ is $\left\{-e_{i} \wedge x\right\}_{i=1 \ldots 3}$ where $e_{i}$ is the $i^{t h}$ unit vector. Thus, the vector field can always be written

$$
f(x, t)=-\lambda(x, t) \wedge x
$$

for some vector function $\lambda$. Consequently, it must be possible to rewrite the observer (1.28) as

$$
\dot{\hat{x}}=\Omega(t) \hat{x}-v(t, \hat{x}, y) \wedge \hat{x} .
$$

Indeed, this is the case as the following simple calculation shows

$$
\begin{aligned}
\left(I-\hat{x} \hat{x}^{\prime}\right)(y-\hat{x}) & =\left(I-\hat{x} \hat{x}^{\prime}\right) y=y-\hat{x}\left(\hat{x}^{\prime} y\right)=y \hat{x}^{\prime} \hat{x}-\left(\hat{x} y^{\prime}\right) \hat{x} \\
& =\left(y \hat{x}^{\prime}-\hat{x} y\right) \hat{x}=-(y \wedge \hat{x}) \wedge \hat{x}
\end{aligned}
$$

Apparently, the projected Kalman filter can be written as

$$
\dot{\hat{x}}=\Omega(t) \hat{x}-l(t)(y \wedge \hat{x}) \wedge \hat{x}
$$

which will be referred to as the geometric attitude observer. The geometric interpretation is depicted in Figure 1.2. Ideally, $y=x=\hat{x}$, and then $y \wedge x=0$ so the estimate evolves according to a copy of the underlying systems dynamics $\dot{x}=\Omega(t) x$. If not, then a corrective angular velocity is added, driving $\hat{x}$ towards $y$. This angular velocity is directed along the vector $y \wedge \hat{x}$. 


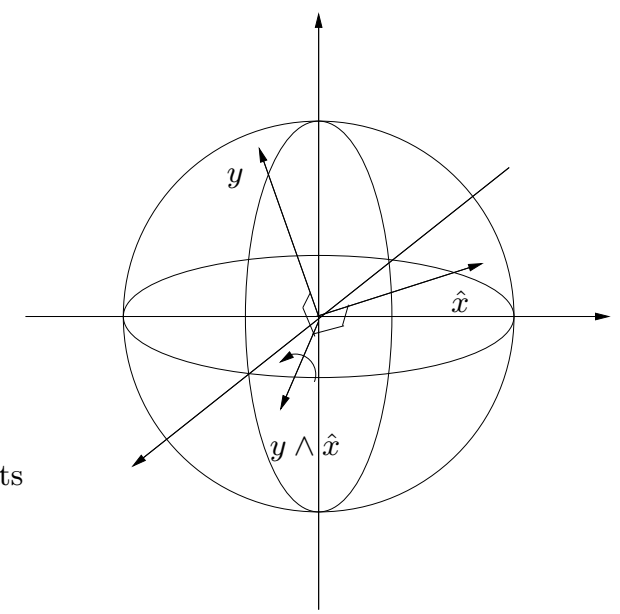

Fig. 1.2. Geometric interpretation of the projected Kalman filter

\subsubsection{Acceleration handling}

If the acceleration induced noise is modeled well by white noise then it is straightforward to apply either of the two filters presented with $q_{2}(t)=q_{2}$, the covariance of the acceleration noise. For a mobile robot such as a walking one, the acceleration pattern will typically consist not only of white noise but also of impulse like signals when the feet hit the ground. Other applications where non-white accelerations may occur is airplanes, cars or trains. When these vehicles turn, the centripetal acceleration will typically be non-white. Whatever characteristics the acceleration have, it might be argued that one may choose $q_{2}$ so high that the errors in the worst case scenario are small enough. The problem with this approach is that it is conservative and the filter performance will be unnecessarily bad. In an attempt to handle the problem of non-white accelerations we propose to use a variable $q_{2}=q_{2}(y, \hat{x})$ that is a function of some acceleration estimate. We propose two different approaches, one switching approach and one continuous approach. In both cases the following structure

$$
q_{2}(t)=(1+\phi(y, \hat{x})) \hat{q}_{2}
$$

where $0 \leq \phi(y, \hat{x})<\infty$ is used. In the following we only consider the scalar version of the filters but the same line of thinking can be applied to the general case. The different approaches presented below are equally applicable to the two filters. For both methods, a reliable acceleration estimate is of course necessary.

The most natural acceleration estimate is

$$
\hat{w}=y-\hat{x}
$$


as $y=x+w$ and in steady state, ideally $x=\hat{x}$. The question is now whether this estimate can safely be used or if there are false indications of low accelerations? Are there unfortunate combinations of $x, \hat{x}$ and $w$ such that $\hat{w}$ is small even if $w$ is not? The answer is yes. Consider the equation $\|\hat{w}\|=0$. As

$$
\|\hat{w}\|=\|x+w-\hat{x}\|
$$

we have a false indication of zero acceleration if $w=\hat{x}-x$. This situation is depicted for a planar case in Figure 1.3. If $x=\hat{x}$ then there are no false

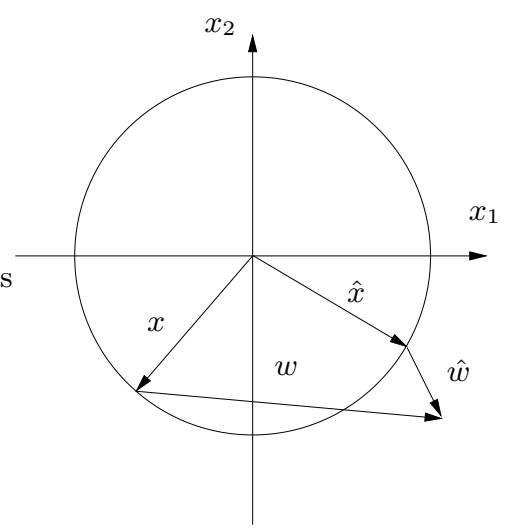

Fig. 1.3. False indications of low acceleration.

indications and for small differences $\hat{x}-x$ the acceleration must be small. Therefore, once the observer have converged, false indications will not be so fatal to performance. What though may happen is that combinations of state, state estimate and acceleration may prevent the observer from converging. However unlikely, it still must be considered. It should though be noted that in a typical robotics application, the robot would start from a stand still position with no acceleration. The observer can then be allowed to converge before the actual movement starts. To reduce the risk of false indications, the following time-window based acceleration norm estimate may be used

$$
\widehat{\|w\|}(t)=\max _{\tau \in\left[t-T_{0}, t\right]}\|y(\tau)-\hat{x}(\tau)\| .
$$

Here $T_{0}$ is a parameter that needs to be tuned. The higher it is chosen the more certain it is that no false indications take place, but, the filter design is more conservative. It should be pointed out that a related idea has been proposed earlier by Foxlin $([8],[9])$. In Figure 1.4, an illustrative example of $\widehat{\|w\|}$ and $\|y-\hat{x}\|$ shows how the expression (1.46) excludes the spurious occasions where $y-\hat{x}=0$ but captures the longer phases of $y-\hat{x}=0$. 


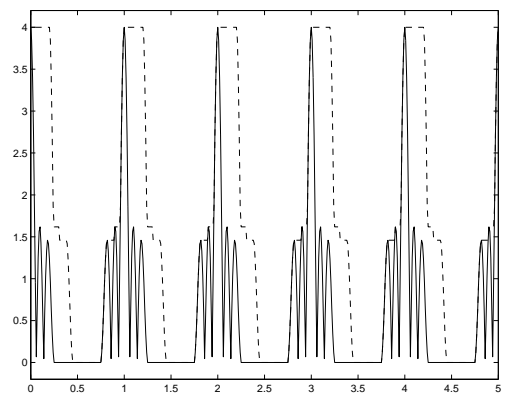

Fig. 1.4. Time-window approach for avoiding false detections

If the accelerations are well modeled as consisting of phases of low acceleration and of phases of high acceleration, then a reasonable idea is to use a switching structure where

$$
\phi(y, \hat{x})=\left\{\begin{array}{l}
0, \widehat{\|w\|}=0 \\
\bar{\phi}, \widehat{\|w\|} \neq 0
\end{array}\right.
$$

Care need though to be taken when using this switching structure. The parameters in the Riccati equation (1.20) are now changing discontinuously. So is the observer vector field. How to prove stability for switching and hybrid systems is an open issue [5] and indeed, in the paper [6] where bounds on the Riccati equation is shown, the involved matrices are assumed to be continuous. Fortunately, these problems can be avoided by starting with a sampled-data model of (1.3) and working in discrete time. This approach is described by Rehbinder and $\mathrm{Hu}$ in [19].

In an environment where the acceleration is not well modeled as composed by high/low accelerations, a continuously tuned acceleration noise parameter may be used. Here we propose

$$
\phi(y, \hat{x})=\alpha^{2} \widehat{\|w\|}^{2}
$$

where $\alpha$ is a parameter that needs to be tuned. To avoid the technical difficulties with the switching structure (1.47) we study the continuous approach (1.48). Further, our main application is walking robots and we argue that a switching structure is less suitable than the continuous structure (1.48). In the following experimental evaluation, the acceleration estimate (1.46) has not been used.

\subsection{Summary and discussion}

In this paper we have presented an approach to solving the attitude estimation problem for a rigid body. The algorithm does in a theoretically consistent 
and easily implementable way solve this problem. The application in mind is a walking robot but the algorithms are in no way dependent on that application and could just as well be applied to other robots such as flying or climbing ones. For climbing robots, the use of the global representation instead of the more standard yaw/pitch/roll parameterization could prove very useful as such robots usually have an attitude out of the bounds for which that parameterization is valid. Further, the kinematics representation is very important as the linear Kalman filter can be used which makes it possible to easily obtain theoretical convergence results. We are also able to formulate a nonlinear observer that evolves on the sphere. It is worth emphasizing that the hard nonlinear problems often associated with the problem studied in this paper originate from the choice of coordinates and that it was possible to avoid them by a suitable representation. The observers presented have also been evaluated in experiments. The most challenging and interesting research topic that should follow this study is to study observer design for systems on Lie groups in a more general setting. Questions to ask are: Is it possible to find conditions guaranteeing that projected Kalman filters in some more general setting are convergent? Does the geometric attitude filter have some generality? Are there any fundamental connections to the observer presented in [16], which has a similar structure? From the applications perspective, more careful experimental testing is necessary. A discrete-time counterpart of the pseudo-linear Kalman filter, presented in [19], has been used in feedback loops for walking robot balancing. The next experimental step is to close the loop with the observers presented in this paper.

\section{References}

1. M. A. Abidi and R. C. Gonzales, editors. Data Fusion in Robotics and Machine Intelligence. Academic Press, 1992.

2. A. J. Baerveldt and R. Klang. A low-cost and low-weight attitude estimation system for an autonomous helicopter. In IEEE International Conference on Intelligent Engineering Systems, Proceedings, pages 391-395, 1997.

3. J. Balaram. Kinematic observers for articulated rovers. In Proceedings of the 2000 IEEE International Conference on Robotics and Automation, pages 25972604, San Francisco, April 2000.

4. B. Barshan and H. F. Durrant-Whyte. Inertial navigation systems for mobile robots. IEEE Transactions on Robotics and Automation, 11(3):328-342, June 1995.

5. M. S. Branicky. Multiple Lyapunov functions and other analysis tools for switched and hybrid systems. IEEE Transactions on Automatic Control, 43(4):475-482, April 1998.

6. R. S. Bucy. The Ricatti equation and its bounds. Journal of computer and system sciences, 6:343-353, 1972.

7. F. Bullo and R. M. Murray. Tracking for fully actuated mechanical systems: A geometric framework. Automatica, 35(1):17-34, 1999. 
8. E. Foxlin. Inertial head-tracker sensor fusion by a complementary separetebias Kalman filter. In Proceedings of the IEEE 1996 Virtual Reality Annual International Symposium, pages 185-94, 1996.

9. E. Foxlin, M. Harrington, and Y. Altshuler. Miniature 6-DOF inertial system for tracking HMD. In Proceedings of the SPIE, volume 3362, pages 214-228, 1998.

10. M. Greene. A solid state attitude heading reference system for general aviation. In Proceedings.1996 IEEE Conference on Emerging Technologies and Factory Automation, volume 2, pages 413-17, 1996.

11. J.Ingvast, C. Ridderström, F. Hardarson, and J. Wikander. Improving a trotting robot's gait by adapting foot reference offsets. In Int. Conf. on Climbing and Walking Robots, Karlsruhe,Germany, September 2001.

12. D. E. Koditschek. The application of total energy as a Lyapunov function for mechanical control systems. In J. E. Marsden, P. S. Krishnaprasad, and J. C. Simo, editors, Dynamics and Control of Multibody Systems, volume 97, pages 131-157. AMS, 1989.

13. E. Lefferts, F. Markley, and M. Shuster. Kalman filtering for spacecraft attitude estimation. Journal of Guidance, Control and Dynamics, 5(5):417-429, SeptOct 1982

14. A. M. Madni, D. Bapna, P. Levin, and E. Krotkov. Solid-state six degree of freedom, motion sensor for field robotic applications. In Proceedings 1998 IEEE/RSJ International Conference on Intelligent Robots and System, volume 3, pages 1389-9, 1998.

15. H. Rehbinder and B. K. Ghosh. Pose estimation using line based dynamic vision and inertial sensors. Submitted to the IEEE Transactions on Automatic Control.

16. H. Rehbinder and B. K. Ghosh. Rigid body state estimation using dynamic vision and inertial sensors. In Proceedings of the 40th IEEE Conference on Decision and Control, 2001. (To appear).

17. H. Rehbinder and X. Hu. Nonlinear pitch and roll estimation for walking robots. In Proceedings of the 2000 IEEE International Conference on Robotics and Automation., volume 3, pages 2617-2622, San Francisco, CA, USA, 2000.

18. H. Rehbinder and X. Hu. Nonlinear state estimation for rigid body motion with low-pass sensors. Systems \& Control Letters, 40(3):183-190, 2000.

19. H. Rehbinder and X. Hu. Drift-free attitude estimation for accelerated rigid bodies. In Proceedings of the 2001 IEEE International Conference on Robotics and Automation, volume 4, pages 4244-4249, Seoul, Korea, 2001.

20. C. Ridderström, J. Ingvast, F. Hardarson, M. Gudmundsson, M. Hellgren, J. Wikander, T. Wadden, and H. Rehbinder. The basic design of the quadruped robot Warp1. In International Conference on Climbing and Walking Robots, Madrid, Spain, October 2000.

21. T. Sakaguchi, K. Tsutomu, H. Katayose, K. Sato, and S. Inokuchi. Human motion capture by integrating gyroscopes and accelerometers. In Proceedings of the 1996 IEEE/SICE/RSJ International Conference on Multisensor Fusion and Integration for Intelligent Systems, pages 470-475, 1996.

22. R. Smith, A. Frost, and P. Probert. Gyroscopic data fusion via a quartenionbased complementary filter. In Proccedings of the SPIE - The International Society for Optical Engineering. vol.306\%, pages 148-59, 1997.

23. S. Soatto, R. Frezza, and P. Perona. Motion estimation via dynamic vision. IEEE Transactions on Automatic Control, 41(3):393-413, March 1996. 
24. E. D. Sontag. Mathematical Control Theory : Deterministic Finite Dimensional Systems. Springer, New York, 1998.

25. J. Vaganay, M. Aldon, and A. Fournier. Mobile robot attitude estimation by fusion of inertial data. In Proceedings of the 1993 IEEE International Conference on Robotics and Automation, volume 1, pages 277-82, 1993.

26. P. K. Varshney, editor. Proceedings of the IEEE, Special Issue on Data Fusion, volume 85. IEEE, January 1997. 